\title{
SAPF for the Inter-harmonics on Grid Network Compensation
}

\author{
Chao Zhang ${ }^{1}$ and Yi J. Zhang ${ }^{2}$ \\ ${ }^{1}$ China University of Mining and Technology, Xuzhou, China \\ ${ }^{2}$ Longkou Mineral Group Co. Ltd, Longkou, China \\ E-mail:lunwenzzz@163.com
}

\begin{abstract}
Using SAPF (shunt active power filter) has been proved as an effective way for harmonic compensation. In practical applications, the performance of SAPF will be impacted by interharmonics and non-periodic disturbances. This paper analyzed the CPT (conservative power theory) definition of power theory and the application method for SAPF. Repetitive controller and PI controller were used as a combination way for the harmonic and inter-harmonic compensation. By the EID (equivalent input-disturbance) controller, the performance for non-periodic interference suppression of SAPF can be improved. By simulation with MATLAB, the models of SAPF system, repetitive controller and EID controller were built. Through the computer simulation method, we can verify the designed system performance.
\end{abstract}

Keywords: conservative power theory (CPT), equivalent input-disturbance (EID)

\section{Introduction}

Inter-harmonic means that harmonic component which frequency is not equal to the fundamental and multiple fundamental frequencies. With the application of HVDC equipment, electric arc furnaces, converters and other power electronic equipments, inter-harmonics were produced [1,2]. Inter-harmonics have similar hazards with harmonics. More seriously, they also can cause voltage flicker and impact torque [3]. As frequencies of inter-harmonics have always been changed with different operating conditions of harmonic source equipments, inter-harmonics is difficult to be accurately detected and eliminated.

SAPF has been widely used to resolve harmonic pollution issues. For the design of SAPF, different grid environments and load requirements should be considered. SAPF should be able to run at different complex grid environments, and do not make interference to the grid. At present, the research focuses of inter-harmonic are on the detection method. The harmonic suppression method is mainly used with passive filters. The way that using SAPF to eliminate inter-harmonic content was less studied. And existing harmonic detection and control methods of SAPF are not effective for inter-harmonics environment [4]. So, it is significant to do the research for the inter-harmonics and harmonics detection and compensation methods based on SAPF.

Currently, detection methods of harmonics and inter- harmonics can be divided into two categories: frequency- domain and time-domain. Harmonic current can be obtained by frequency-domain detection methods. But these methods are characterized by large amount of calculation, and poor real-time performance. The existing time-domain detection methods are based on non-sinusoidal power theory, and they are applicable for SAPF. These methods are simple in structure, and they are widely used. There are two types of commonly used timedomain detection methods which based on the power theory, the instantaneous power theory and the Fryze power theory. The harmonic current cannot be accurately detected by method with traditional instantaneous reactive power theory ( $p-q$ theory) when three-phase voltage is 
unbalance and waveform is distorted. Detection method based on p-q-r power theory, of which the zero power is defined [14], can be used for the detection of three-phase unbalanced system. Harmonic and reactive current detection problems can be solved by universal instantaneous power theory and its improved algorithm when the three-phase voltage is unbalance and waveform is distorted [15, 16]. FBD method was proposed by Fryze, and improved by Buchholz and Dpenbrock. By separating the current with concept of equivalent conductance, the physical meaning of the current component was discussed. And compared to the traditional instantaneous power definition, without coordinate transformation, FBD method was characterized by a relatively simple algorithm $[17,18]$. The instantaneous power was defined by CPT (conservative power theory) in the three phase unbalance and distortion system [19]. Compared to the p-q power theory and FBD power theory, the unbalanced and distortion current is more accurately expressed by CPT theory [20, 21]. But it needs further study for the grid with inter-harmonics situation.

The periodic reference signal or the periodic interference signal will be controlled by repetitive control method. And when it combined with PI control, or predictive control, the PWM converter can get better control performance for current. But repetitive control performance for non-periodic signal or non-periodic interference signal is poor. Therefore, it is important to improve the control system for better non-periodic control performance. There are two ways to improve the performance, HORC (high-order repetitive controller) [22] and adaptive repetitive controller [23]. Though the control performance can be improved by these methods, the complexity of the system will be increased, and it will be difficult to achieve system stability. If we consider the non-periodic signals as interference, the disturbance observer can be used to improve the non-periodic control performance of repetitive controller. EID (equivalent input disturbance) observer [24] is different with the usual disturbance observer, which is not based on the inverse system theory, but based on an active disturbance rejection method. This method is simple to implement and can be used for repeatedly controller to improve the non-periodic disturbance rejection performance.

In this paper, the model of three-level SAPF was built. SAPF directive harmonic current detection method under the inter-harmonic environment based on the definition of CPT power theory was studied. Both the repetitive control and PI control was used for the grid current harmonics and inter-harmonics compensation. By introducing EID controller improves the SAPF control performance for non-periodic signal interference. Analysis of the designed system stability and sensitivity of the issue, gives a suitable inter-harmonics environment SAPF system design methodology. Simulation and experiment show that the designed system can accurately detect harmonic, inter-harmonic content, effective on the grid to compensate harmonics and inter-harmonics.

\section{Mathematic Model of Three-level SAPF on Virtual Flux Oriented}

The main circuit topology of SAPF is a diode-clamped three-level inverter. Three kinds of switch state can be obtained by four switches of each phase leg from threelevel converter, which is shown in Figure 1 (a). 


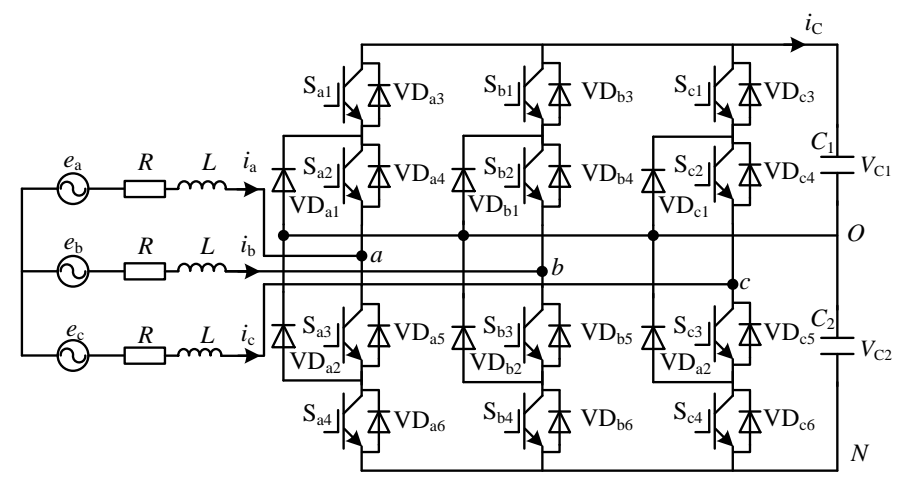

Figure 1. The Topology of Three-level Converter

According to Kirchhoff law and the structure shown in Fig.1, state-space expression of SAPF in dq reference frame is given directly by:

Where,

$$
Z \dot{X}=A X+B e
$$

$$
\begin{aligned}
& Z=\operatorname{diag}\left[\begin{array}{llll}
L & L & C_{1} & C_{2}
\end{array}\right] \\
& X=\left[\begin{array}{llll}
i_{d} & i_{q} & V_{C 1} & V_{C 2}
\end{array}\right]^{\mathrm{T}}, B=\operatorname{diag}\left[\begin{array}{llll}
1 & 1 & 0 & 0
\end{array}\right] \\
& e=\left[\begin{array}{llll}
0 & \sqrt{\frac{3}{2}} V_{m} & 0 & 0
\end{array}\right]^{\mathrm{T}} \\
& A=\left[\begin{array}{cccc}
-R & \omega L & -S_{d 1} & S_{d 2} \\
-\omega L & -R & -S_{q 1} & S_{q 2} \\
S_{d 1} & S_{q 1} & 0 & 0 \\
-S_{d 2} & -S_{q 2} & 0 & 0
\end{array}\right]
\end{aligned}
$$

\section{The Inter-harmonic Detection Method Based on CPT Theory}

\subsection{The CPT Framework}

CPT theory, which has been put forward in recent years, is power definition method under the nonlinear time-domain condition. For a continuous variable $x(t)$, over period $T$, the original function and derivative function can be defined as follows:

$$
x^{\prime}(t)=\int_{0}^{t} x(\tau) \mathrm{d} \tau ; x^{\prime \prime}(t)=\frac{\mathrm{d}}{\mathrm{d} t} x(t)
$$

The DC component is defined as:

$$
\bar{x}^{\prime}=\frac{1}{T} \int_{0}^{T} x^{\prime}(t) \mathrm{d} t
$$

Considering $\omega=2 \pi / T$, homo-variable of $x$ can be defined as:

$$
\hat{x}=\omega\left(x^{\prime}-\bar{x}^{\prime}\right) ; \breve{x}=\frac{1}{\omega} x^{\prime \prime}
$$

Note that $\hat{x}$ and $x$ are dimensionally homogeneous to $x$, and they are equal in the amplitude of the resultant signals, so we called $x, \bar{x}$ and $\bar{x}$ the homo-variables. According to the definition of CPT theory, for three-phase system,

Active power $P$ is defined as:

$$
P=\langle u, i\rangle=\frac{1}{T} \int_{0}^{T} u(t) \cdot i(t) \mathrm{d} t
$$


Reactive power $Q$ is defined as:

$$
Q=\langle\hat{u}, i\rangle=\frac{1}{T} \int_{0}^{T} \hat{u}(t) \cdot i(t) \mathrm{d} t
$$

Active current $i_{\mathrm{a}}$ is defined as:

$$
i_{a}=\frac{P}{\|u\|^{2}} u=G_{a} \cdot u
$$

Reactive current $i_{\mathrm{r}}$ is defined as:

$$
i_{r}=\frac{Q}{\|\hat{u}\|^{2}} \hat{u}=B_{r} \cdot \hat{u}
$$

Void current $i_{\mathrm{v}}$ is defined as:

$$
i_{v}=i-i{ }_{a}{ }^{-i} i_{r}
$$

\subsection{The Inter-harmonic Detection Method based on CPT Theory}

In order to use the CPT theory to detect SAPF three-phase harmonic current, assuming three-phase sinusoidal and balance system, with the initial phase of supply voltage which equals to 0 , per unit voltage can be expressed as:

$$
\left\{\begin{array}{c}
e_{a}=\sin (\omega t) \\
e_{b}=\sin \left(\omega t-\frac{2 \pi}{3}\right) \\
e_{c}=\sin \left(\omega t+\frac{2 \pi}{3}\right)
\end{array}\right.
$$

Where, $e_{\mathrm{a}}, e_{\mathrm{b}}$ and $e_{\mathrm{c}}$ are three phase per unit supply voltage, and $\omega$ is the angular frequency. According to CPT definition, homo-variable of ea can be expressed as:

$$
\left\{\begin{array}{l}
\hat{e}_{a}=-\cos (\omega t) \\
\check{e}_{a}=\cos (\omega t)
\end{array}\right.
$$

The three-phase load current is given by:

$$
\left\{\begin{array}{c}
i_{a}=\sum_{h=1}^{\infty}\left[I_{h}^{+} \sin \left(h \omega t-\theta_{h}^{+}\right)+I_{h}^{-} \sin \left(h \omega t-\theta_{h}^{-}\right)+I_{h}^{0} \sin \left(h \omega t-\theta_{h}^{0}\right)\right. \\
i_{b}=\sum_{h=1}^{\infty}\left[I_{h}^{+} \sin \left(h \omega t-\frac{2 \pi}{3}-\theta_{h}^{+}\right)+I_{h}^{-} \sin \left(h \omega t+\frac{2 \pi}{3}-\theta_{h}^{-}\right)+I_{h}^{0} \sin \left(h \omega t-\theta_{h}^{0}\right)\right. \\
i_{c}=\sum_{h=1}^{\infty}\left[I_{h}^{+} \sin \left(h \omega t+\frac{2 \pi}{3}-\theta_{h}^{+}\right)+I_{h}^{-} \sin \left(h \omega t-\frac{2 \pi}{3}-\theta_{h}^{-}\right)+I_{h}^{0} \sin \left(h \omega t-\theta_{h}^{0}\right)\right.
\end{array}\right.
$$

Where, ia, ib and ic are the three-phase load current. I is the amplitude of the harmonic current. $\theta$ is the initial phase angle of the harmonic current. Subscript $h$ is the harmonic number. When $\mathrm{h}=1$, it indicates the fundamental component; when $\mathrm{h}$ is integer, it means multiple fundamental frequency components; when $\mathrm{h}$ is non-integer, it indicates the harmonic components. Superscript + , - and 0 represent positive, negative and zero sequence component.Greater view, the artificial fish could find global extremum and be convergent more easily. The greater of artificial fish's moving step, the optimization process converged faster. If the problem with local extrema was not very prominent, increasing the number of attempt times could reduce the artificial fish random walk, and improve convergence efficiency. When the case with prominent local extrema was serious, reducing the attempt times of prey could increase the probability of the artificial fish random moving, and overcome the impact of local extrema. Therefore, the artificial fish adjusted view, moving step and times of prey attempt automatically according to the following equation (1),(2) and 
(3). It was conducive for success of artificial fish's prey and the algorithm performance improvement. The artificial fish's prey behavior with adjusted parameter was called adaptive enhanced prey behavior, which was helped to improve the probability of prey success [12].

According to CPT definition, taking into the voltage and current expressions, it can be calculated as:

$$
P=\frac{1}{T} \int_{0}^{T} i(t) e(t) \mathrm{d} t=\frac{1}{T} \int_{0}^{T} i(t) \sin \omega t \mathrm{~d} t
$$

Taking equation (4) into equation (1), active current can be calculated as:

$$
i_{a}(t)=\frac{P}{\left\|e_{\Sigma}\right\|^{2}} e=\frac{\frac{1}{T} \int_{0}^{T} i(t) \sin \omega t \mathrm{~d} t}{(1 / \sqrt{2})^{2}} \sin \omega t=\frac{2}{T} \int_{0}^{T} i(t) \sin \omega t \mathrm{~d} t \cdot \sin \omega t
$$

According to CPT theory, homo-variables of three-phase supply voltage can be defined as:

$$
\left\{\begin{array}{c}
\hat{e}_{a}=-\cos (\omega t) \\
\hat{e}_{b}=-\cos \left(\omega t-\frac{2 \pi}{3}\right) \\
\hat{e}_{c}=-\cos \left(\omega t+\frac{2 \pi}{3}\right)
\end{array}\right.
$$

According to the reactive power definition of CPT theory, instantaneous reactive power $Q$ can be obtained as:

$$
Q=\frac{1}{T} \int_{0}^{T} i(t) \hat{e}(t) \mathrm{d} t=-\frac{1}{T} \int_{0}^{T} i(t) \cos \omega t \mathrm{~d} t
$$

Taking equation (5) into equation (2), reactive current can be calculated as:

$$
i_{r}(t)=\frac{Q}{\left\|\hat{e}_{\Sigma}\right\|^{2}} e=\frac{\frac{1}{T} \int_{0}^{T} i(t) \cos \omega t \mathrm{~d} t}{(1 / \sqrt{2})^{2}} \cos \omega t=\frac{2}{T} \int_{0}^{T} i(t) \cos \omega t \mathrm{~d} t \cdot \cos \omega t
$$

Note that equation (4) corresponds with the fundamental current active component. And equation (6) corresponds with the fundamental current reactive component. Taking the equation (4) and equation (6), the fundamental component can be got by the addition of the two final equations. Besides the fundamental positive sequence component, the rest components are the SAPF harmonic detection signal. Based on CPT power definition, SAPF harmonic detection system is shown in Figure 2.

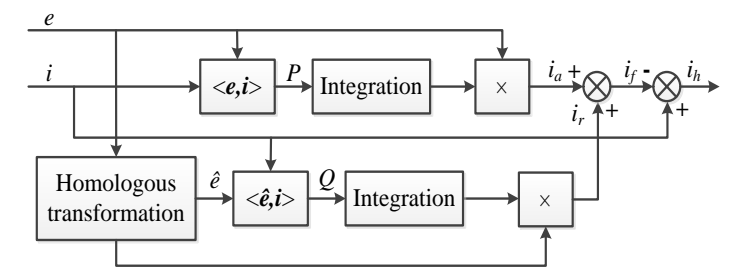

\section{Figure 2. Harmonic Detection System based on CPT}

Considering ip-iq detecting method based on instantaneous reactive power theory as a comparison, the grid inter-harmonic detection effect of the system, which was shown in Figure 2, can be verified by simulation. The fundamental frequency of testing wave was $50 \mathrm{~Hz}$. The harmonic components which frequencies were $80 \mathrm{~Hz}$ and $250 \mathrm{~Hz}$, were added at Time $=0.08 \mathrm{~s}$. The simulation results were shown in Figure 3. 
The waveform ia and the fundamental detection waveforms which detected by ip-iq method and CPT method, were shown in the Figure 3 (a). Note that, before adding the harmonic component, the fundamental detection waveform got by CPT method can coincide with ia. But for the waveform got by ip-iq method, there is a certain phase offset, which is caused by the coordinate transformation of the instantaneous power theory [25]. Subtracted the fundamental detection waveform and ia, the harmonic component can be obtained, which was shown in Figure 3 (b). It can be shown as that, since there is phase offset phenomenon, the amplitude of harmonic component detected by ip-iq method was finally amplified.

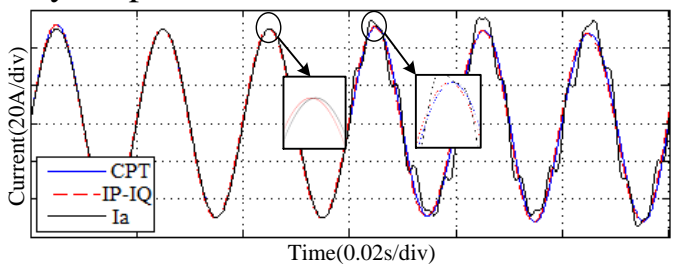

(a) Fundamental detection waveform

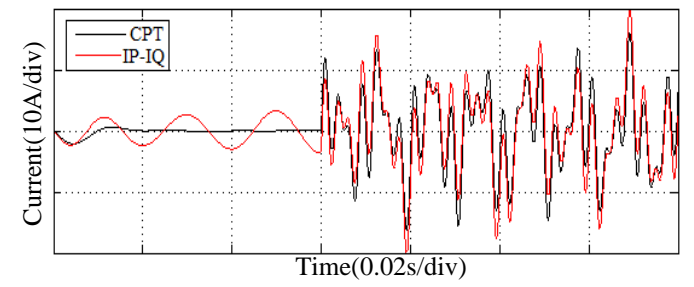

(b) Harmonic detection waveform

\section{Figure 3. Comparison of current waveforms}

\section{Compensation System based on EID and Repetitive Controller}

\subsection{Influence of Inter-harmonics}

In order to improve the compensation effect of the SAPF, detection accuracy of SAPF should be improved. In addition, it is also necessary to improve the performance of SAPF compensation controller. Currently, many of the repetitive controllers were used for compensation according to command signal detected. To simplify the analysis, a repetitive controller was given:

$$
G_{\mathrm{RE}}(z)=\frac{1}{1-z^{-N}}
$$

Where, $N=T / T_{\mathrm{s}}, T$ is the period of the command signal, $T_{\mathrm{s}}$ is the sampling period. The frequency characteristic was shown in Figure 4.

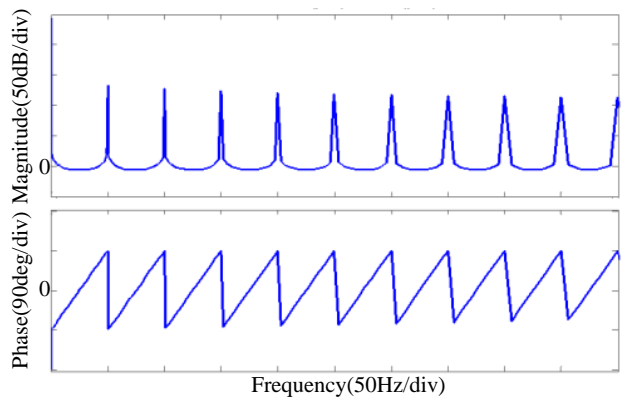

Figure 4. Bode Diagram of Repetitive Controller 
As we can see from the Figure 4, with the fundamental frequency and its multiples, amplitude gains are infinite and phases are all zero, which meet the integrator requirements. So the no static error track of harmonics could be achieved. But it also can be seen that with the non-integer multiples of the fundamental frequency, the frequency characteristic of the repetitive controller is not ideal. So under the interharmonic environment, satisfactory control effect cannot be got simple with repetitive controller. It should be combined with other control methods to realize SAPF control.

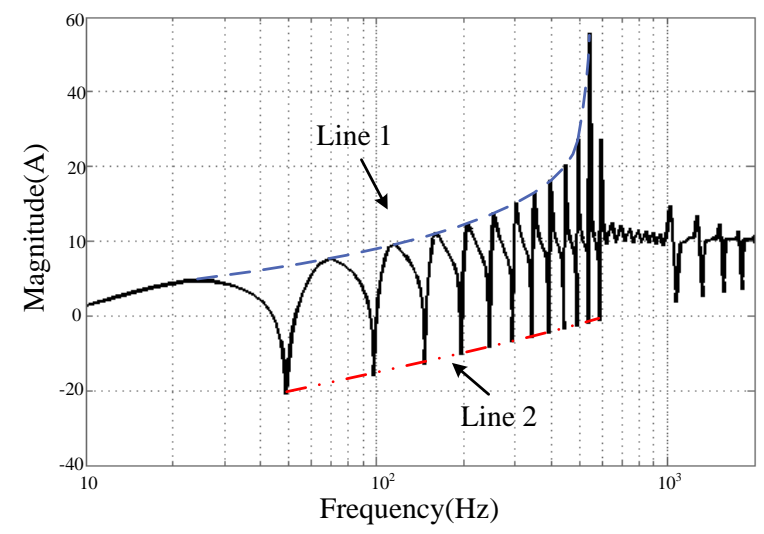

Figure 5. Amplitude-frequency of Traditional Repetitive Controller

As it was shown in Figure 5, the amplitude-frequency curve of traditional repetitive controller sensitivity function was got. According to Figure 5, with fundamental frequency $f=50 \mathrm{~Hz}$ and its integer multiple frequencies, the conventional repetitive controller got better performance for disturbance suppression, as it was shown in Figure 5 line 2. But at other frequencies, the disturbance rejection performance is worse than the former, that is, repetitive controller got worse disturbance rejection performance for non-integer multiple frequencies, which was shown in Figure 5 line 1. This phenomenon can be explained by the Bode integral theorem. For linear time-invariant systems, with the control performance of one band improved by the controller design, the inevitable control performance deterioration of the system in another band will got. So it is necessary that during control system design, the band with deterioration performance should be disposed in the signal band with little impact on system.

In order to solve the problem that inter-harmonics detection accuracy is not enough and compensation effect will get worse by the repetitive controller for inter-harmonics, the following measures were proposed. Firstly, FBD method was used to eliminate the need for coordinate transformation. It will avoid the inter-harmonic amplification and phase deviation by traditional detection methods. At last, repetitive controller combined with EID controller was used to improve the system amplitude-frequency characteristic in the inter-harmonic frequency.

Complex repetitive control system consists of improved repetitive controller and PI controller, which are connected in parallel. The periodic signal can be controlled by repetitive controller, so the SAPF compensation accuracy will be improved. And nonperiodic signal can be controlled by PI controller, which can also adjust the delay time caused by repetitive controller, so the dynamic performance of SAPF will be improved. The control system is shown in Figure 6 (b). 


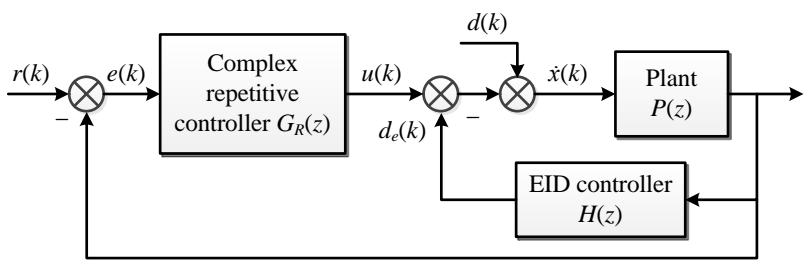

(a) SAPF current compensation control system

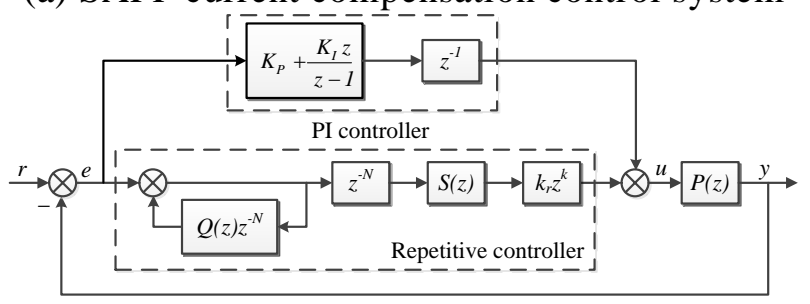

(b) Complex repetitive control system

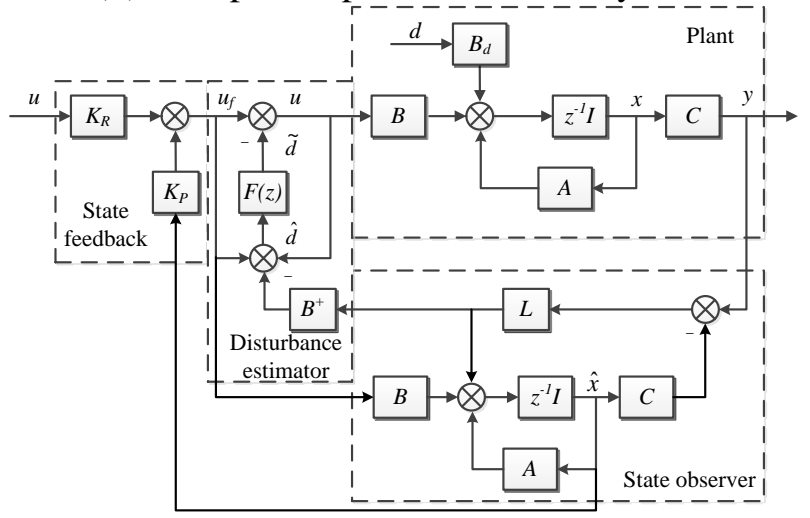

(c) EID control system

Figure 6. The Current Compensation System of SAPF

\subsection{Stability Analysis of Control System}

System stability design is the most basic requirement for control system. Only based on the stable control system, the further system design, considering the rest of the system performance requirements, could be continued.

As it is shown in Figure 6 (a), the control system can be seen as two subsystems which are the complex repetitive PI control system and the EID control system in series. The stability of the control system could be considered as two subsystems both are stable. As control parameters of the composite repetitive control system and the EID control system are without overlap, the two subsystems can be designed individually. For the composite repetitive control system, which is shown in Figure 6 (b), considering the transfer function of the PI controller $G_{\mathrm{PI}}(z)$, the characteristic equation is given by:

$$
\Delta=\left[1+G_{P I}(z) P(z)\right]\left[z^{N}-Q(z)+\frac{k_{r} z^{k} S(z) P(z)}{1+G_{P I}(z) P(z)}\right]
$$

There are two parts in the characteristic equation of complex repetitive control system. It is easy to see that the characteristic equation is $1+G_{\mathrm{PI}}(z) P(z)$, when the system is controlled by PI controller individually. The latter part is the characteristic equation when the repetitive controller adds to the system. Therefore, the stability of 
complex repetitive control system required, based on the stable PI control system, the complex control system is also stable after the addition of repetitive controller.

In order to analyze the stability of the control system, by setting input signal and interference signal of the system shown in Figure 6 (a) to zero, taking the input and output of low-pass filter $F(\mathrm{z})$ in the interference estimator as the system input and output, the EID control system in Fig.6 (c) is equivalent to the system in Figure 7. The component in the dotted-line $G(\mathrm{z})$ is given by:

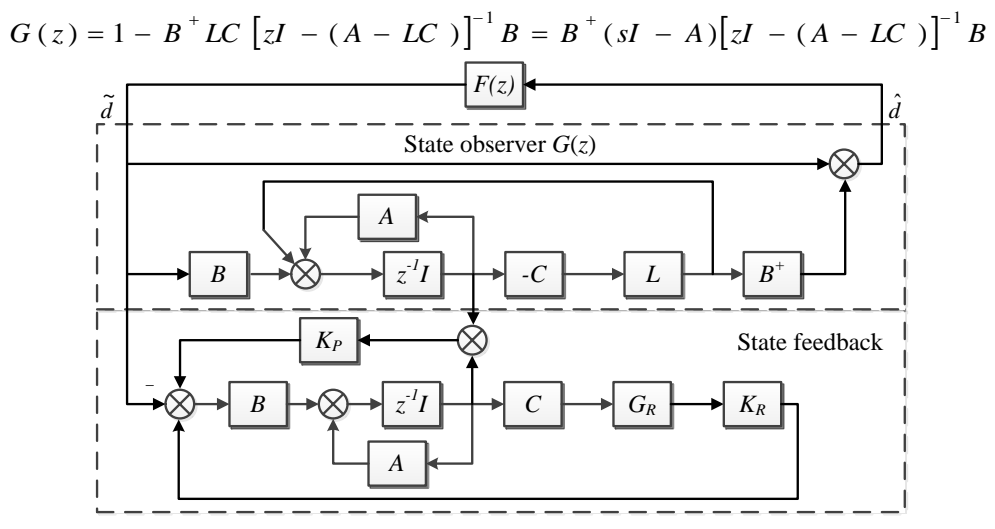

Figure 7. The Equivalent Control System of EID

As it is shown in Figure 7, EID system is divided into three parts, the state observer, the low-pass filter and the state feedback section. As state feedback section will not affect other system stability, it can be designed as the independent part according to the LMI or optimal control method. According to the small gain theory, under the premise that state observer and low-pass filter are stable, it is need to meet that the $\mathrm{H}_{\infty}$ norm of system transfer function is less than 1 for the entire system stability, which means:

$$
\|F(z) G(z)\|_{\infty}<1
$$

\subsection{Design of Control System}

Therefore, the design of observer gain $L$ can be converted into a standard output feedback $\mathrm{H}_{\infty}$ problem. The new input $\omega(\mathrm{t})$ and the new output $z(\mathrm{t})$ were set for the low pass filter $F(\mathrm{~s})$. So the state space expression can be given:

$$
\begin{gathered}
\left\{\begin{array}{c}
\hat{\dot{x}}(t)=A \hat{x}(t)+B \tilde{d}(t)-u(t) \\
z(t)=d(t)-B^{+} u(t) \\
y(t)=C \hat{x}(t)
\end{array}\right. \\
\left\{\begin{array}{c}
\dot{x}(t)=A x(t)+B \omega(t) \\
\tilde{d}(t)=C x(t)
\end{array}\right.
\end{gathered}
$$

For the system was shown in Figure 5 . The $\mathrm{G}$ and $\mathrm{F}$ system can be given as:

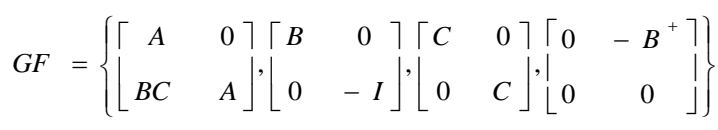

It is need to design output feedback controller $\boldsymbol{u}=\boldsymbol{L} \boldsymbol{y}$ to make equation holds. So we can get $L$ to satisfy that:

$$
\lim _{\delta \rightarrow \infty}[s I-(A-L C)]^{-1} B=0
$$

According to the discussion above, the steps of design can be given as follows: 
Step 1: Design system feedback gain $\mathrm{K}_{\mathrm{p}}, \mathrm{K}_{\mathrm{r}}$;

Step 2: The low pass filter $\mathrm{F}(\mathrm{s})$ should be designed to make equation as follows holds;

$$
\left\{\begin{array}{l}
|F(j \omega)| \approx 1, \quad \omega \leq \omega_{\text {max }} \\
|F(j \omega)|<1, \quad \omega>\omega_{\text {max }}
\end{array}\right.
$$

Where, $\omega_{\max }$ is maximum angular frequency of non-periodic disturbance.

Step 3: Design the observer gain $L$, to make the G(s) stable;

Step 4: Adjust the parameters until the system meets the requirements.

These design steps are very intuitive and easy to understand. The adjustment process of the design is very simple.

\section{Simulation and Experimental Verification}

In order to verify the designed SAPF control performance, based on the Matlab software and three-level experiment platform, simulation and experimental model of SAPF control system was established, which is shown in Figure 8.

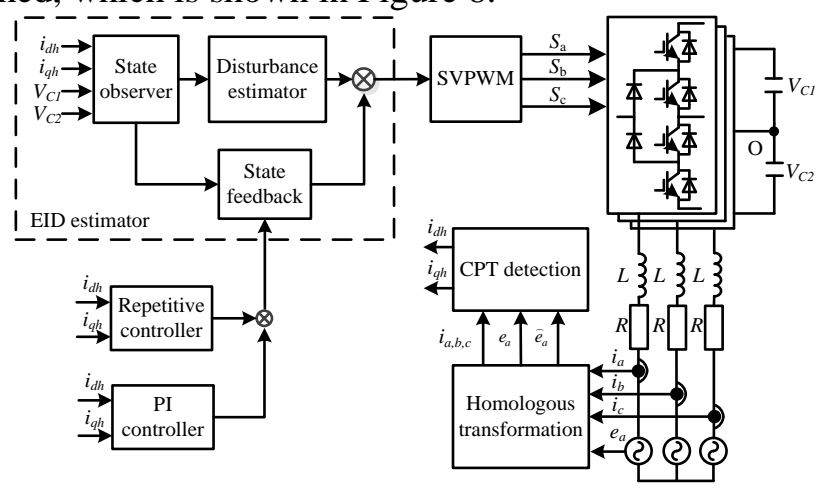

Figure 8. Three-level SAPF Control System Structure

Grid current waveform before the compensation was got in Figure 9 (a). After the compensation by SAPF, the grid current waveform was got in Figure 9 (b). The simulation was made for the two systems with and without the EID controller. Subtracted the compensation current from the detected harmonic current command to obtain the error current signal as it was shown in Figure 9 (c). As it can be seen, the error between compensation current and reference current has become smaller by addition of EID controller. It indicates that by adding EID controller, the better tracking performance can be got for the control system.

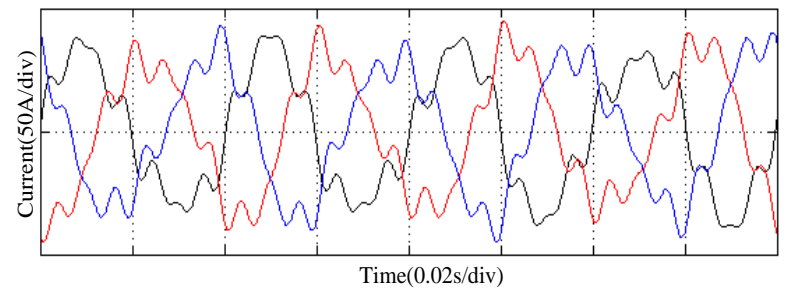

(a) Current waveform before compensation. 


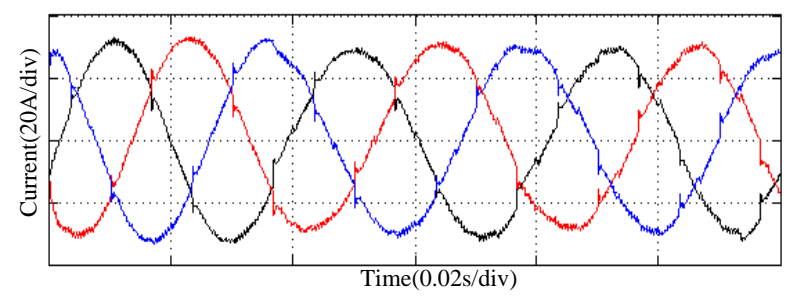

(b) Current waveform after compensation.

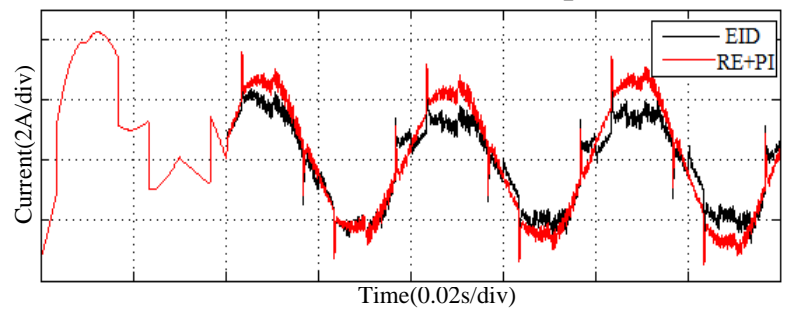

(c) Error between compensation current and reference current.

\section{Figure 9. Comparison of SAPF Harmonic Compensation}

\section{Conclusion}

Given the complex situation of the grid with inter-harmonics, three-level model of shunt active power filter (SAPF) was built. For the harmonics accurate detection, the FBD theory was used to detect the fundamental component of the grid. The interharmonics, parameter fluctuation of APF and the deadband effect were considered as the non-periodic disturbance to the controller. To eliminate the non-periodic disturbance impact on the controller and to improve the control system performance, equivalent input-disturbance (EID) was used based on the traditional repetitive controller. With Matlab and three-level test platform, the SAPF control system was built. The performance of detection and compensation for harmonic and inter-harmonic was verified by the simulation and experiment.

\section{References}

[1] D. H. Zhang, "Study on the phase sequence characteristics of interharmonics," Proceedings of the CSEE, vol. 25, no. 12, (2005) June, pp. 29-34.

[2] D. Basic, "Input current interharmonics of variable-speed drives due to motor current imbalance," IEEE Trans on Power Delivery, vol. 25, no. 4, (2010) October, pp. 2797-2806.

[3] J. Yong, "Research on thyristor conduction angle characteristics in transient process of TCSC," Proceedings of the CSEE, vol. 28, no. 31, (2008) November, pp. 88-93.

[4] W. Zhao, "Influence of non-integer harmonics on HAPF and resolution," Proceedings of the CSEE, vol. 28, no. 12, (2008) April, pp. 73-78.

[5] C. Hsiung, "Inter-harmonic identification using group- Harmonic weighting approach based on the FFT," IEEE Trans on Power Electronics, vol. 23, no. 3, (2008) May, pp. 1309- 1319.

[6] C. Han, H. J. Liu and D. L. Li, "Application of nonuniformly sampling and least square technique in interharmonic measurement," Proceeding of the CSEE, vol. 29, no. 10, (2009), pp. 109-114.

[7] J. Hui and H. G. Yang, "An approach for harmonic/inter-harmonic analysis based on the odd points interpolation correction," Proceedings of the CSEE, vol. 30, no. 16, (2010), pp. 67-72.

[8] C. Y. Zhao and M. F. He, "A novel method for harmonics measurement using phase information of complex wavelet transform," Proceedings of the CSEE, vol. 25, no. 1, (2005), pp. 38-42.

[9] T. Y. Li, "A new method for power quality detection based on HHT," Proceedings of the CSEE, vol. 25, no. 17, (2005), pp. 52-56.

[10] B. W. Ma, H. J. Liu and L. Zhou, "An improved algorithm of interharmonic spectral estimation based on AR model," Proceedings of the CSEE, vol. 25, no. 15, (2005) September, pp. 79-83. 
[11] B. Feng, Q. Fang and H. Y. Yi, et al. "Harmonic and inter-harmonic parameter estimation algorithm of electric power system based on tri-linear decomposition," Proceedings of the CSEE, vol. 33, no. 15, (2013), pp. 1-7.

[12] Y. H. Zhang, G. B. Jin and T. Y. Li, "A novel approach to interharmonics analysis based on adaptive optimal kernel time-frequency distribution," Proceedings of the CSEE, vol. 26, no. 18, (2006), pp. 84-89.

[13] G. Y. Lu, Q. P. Fang and X. S. Cai, "A method for inter-harmonics analysis based on PSO," Transactions of China Electrotechnical Society, vol. 24, no. 12, (2009), pp. 79-83.

[14] Z. L. Tan, X. Li and J. Chen, "A new control strategy of UPQC by using simplified p-q-r theory," Proceedings of the CSEE, vol. 27, no. 36, (2007), pp. 85-91.

[15] M. H. Wang and H. J. Liu, "A universal definition of instantaneous power and broad-sense harmonic theory," Proceedings of the CSEE, vol. 21, no. 9, (2011), pp. 68-73.

[16] D. H. Chen, S. J. Xie and B. Zhou, "An improved synchronous reference frame method for harmonics and reactive currents detection of active power filters," Proceedings of the CSEE, vol. 25, no. 20, (2005), pp. 6267.

[17] Z. Sun, "Detecting methods of reactive power, harmonic and negative -sequence current in electrified rail way systems," Automation of Electric Power Systems, vol. 27, no. 15, August (2003), pp. 53-57.

[18] Z. Y. Guo, "Current detection for four-phase transmission system based on FBD method," Proceedings of the CSEE, vol. 27, no. 22, (2007) August, pp. 87-93.

[19] P. Tenti and P. Mattavelli, "A time-domain approach to power term definitions under non-sinusoidal conditions," Sixth International Workshop on Power Definitions and Measurements under Non-Sinusoidal Conditions, Milano, Italy, (2003).

[20] H. K. M. Paredes, F. P. Marafao and L. C. P. da Silva, "A comparative analysis of FBD, PQ and CPT current decompositions-Part I: Three-phase three-wire systems", 2009 IEEE Bucharest Power Tech Conference, Bucharest, Romania, (2009).

[21] P. Tenti, H. K. M. Paredes and P Mattavelli, "Conservative power theory, a framework to approach control and accountability issues in smart microgrids," IEEE Trans on Power Electronics, vol. 26, no. 3, (2011) April, pp. 664-673.

[22] G. Pipeleers, "Robust high order repetitive control: optimal performance tradeoffs," Automatica, no. 44, September (2008), pp. 2628-2634.

\section{Authors}

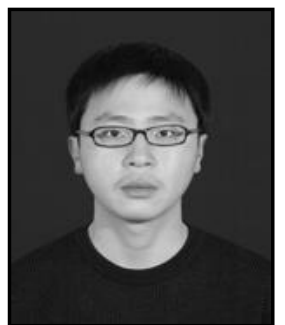

Chao Zhang, He was born in Zou Cheng, Shangdong, China in 1987. He received his B.S. and M.S. from the Shangdong University of Science and Technology, China, in 2008 and 2011, respectively. Since 2011, he has been working towards his Ph.D. in the Department of Information and Electrical Engineering, China University of Mining and Technology. His current research interests include intelligent control, power quality, large capacity SAPF under complex network environment.

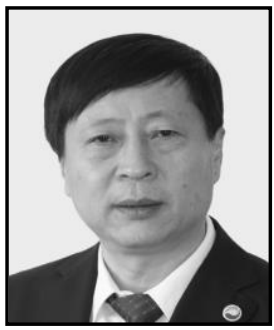

Yi J. Zhang, He was born in Tai An, Shangdong, China in 1961. He received the B.E. degree from the Shangdong University of Science and Technology, China. Now he is working in Longkou Mineral Group Co. Ltd. He is mainly engaged in coal power system security and management. 